\title{
Astrocytoma cells interconnect to resist radiotherapy
}

Cells in astrocytomas form tumour microtubes that interconnect the cells and make them resistant to radiotherapy, according to new research published in Nature.

Understanding the mechanism behind radioresistance could present new therapeutic possibilities.

Using 2-photon microscopy, Frank Winkler and colleagues observed the growth of human glioblastoma cells implanted into mice aged up to 1 year. The researchers saw that glioma cells formed thin, ultralong extensions tumour microtubes - that help them to invade and colonize healthy brain tissue. When the tumours grew, these microtubes formed networks between the astrocytoma cells.

As stated by Winkler, the findings imply that astrocytomas hijack the processes that regulate the formation of neuronal networks in the developing brain. "Our first thought was that it looked like the formation of a new brain within the existing one," Winkler says. "The tumour cells were interconnected in a network that resembles the ones we know of neurons and astrocytes in the healthy brain."

The investigators detected similar tumour microtube networks in resected astrocytomas from patients.
Moreover, the interconnectedness of the glioma cells correlated with tumour malignancy and treatment resistance, suggesting that microtube length could be a biomarker for radioresistance.

Winkler and co-workers discovered that those glioblastoma cells that were interconnected via tumour microtubes were resistant to radiotherapy, the standard treatment for gliomas. Radiosensitivity in glioma cells that are not interconnected with microtubes is thought to result from irradiationinduced dysregulation of intracellular $\mathrm{Ca}^{2+}$ levels. However, Winkler and colleagues discovered that normal $\mathrm{Ca}^{2+}$ levels were maintained in interconnected glioblastoma cells, even after irradiation.

The investigators discovered that the glioblastoma cells can communicate through gap junctions between tumour microtubes, and that this cell-to-cell communication depended on connexin-43 (Cx43). 1p/19q co-deletion - which ablates $\mathrm{Cx} 43$ and strongly correlates with responsiveness to radiochemotherapy - decreased the expression of neuromodulin, resulting in diminished formation of $\mathrm{Cx} 43$ bearing tumour microtubes, and a much better response to radiation.

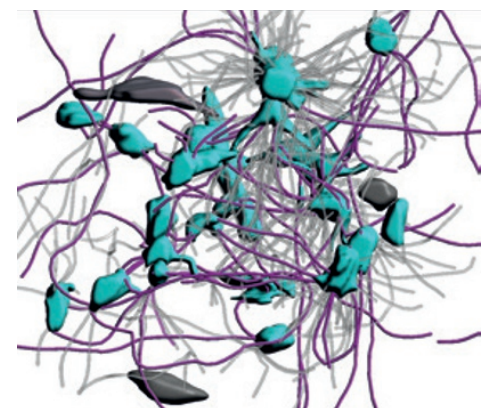

Tumour microtubes (magenta) connect glioblastoma cells (turquoise). Adapted with permission from Macmillan Publishers Ltd $\odot$ Osswald, M. et al. Nature doi:10.1038/ nature16071.

The researchers now plan to investigate whether tumour microtubes are specific to astrocytomas. Moreover, interventions that target molecular machinery that is essential for brain development and formation of tumour microtubes, but is less crucial in the adult brain, could render brain tumours more vulnerable to therapies.

Hemi Malkki

This article was first published in Nature Reviews Neurology doi:10.1038/nrneurol.2015.215

ORIGINAL ARTICLE Osswald, M. et al. Brain tumour cells interconnect to a functional and resistant network. Nature doi:10.1038/nature16071 